\title{
Spin resonances for stored deuteron beams in COSY. Vector polarization. Tracking with Spink
}

\author{
A. U. Luccio, A. Lehrach
}

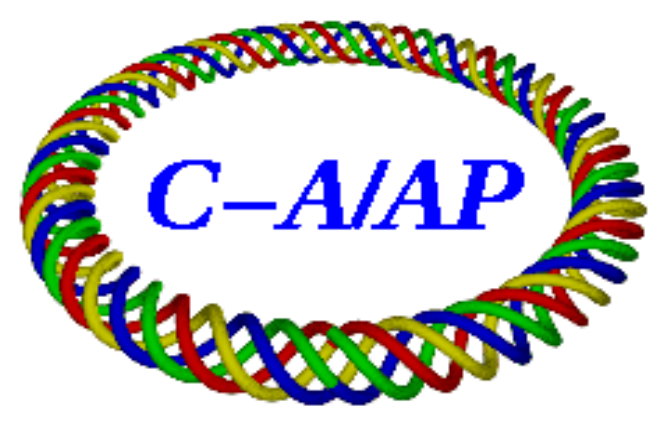

Collider-Accelerator Department Brookhaven National Laboratory Upton, NY 11973

Notice: This document has been authorized by employees of Brookhaven Science Associates, LLC under Contract No. DE-AC02-98CH10886 with the U.S. Department of Energy. The United States Government retains a non-exclusive, paidup, irrevocable, world-wide license to publish or reproduce the published form of this document, or allow others to do so, for United States Government purposes. 


\title{
Spin resonances for stored deuteron beams in COSY. Vector polarization. Tracking with Spink
}

\author{
A.U. Luccio, A.Lehrach
}

April 8, 2008

\begin{abstract}
Results of measureemnts of vector and tensor ploarizatin of a deuteron beam in the storage ring COSY have been published by the SPIN@COSY collaboration[1]. In this experiment a RF Dipole was used that produced spin flip. The strength of the RFD-induced depolarizing resonance was calculated from the amount of spin flipping and the results shown in the figures of the cited paper. In this note we present the simulation of the experimental data (vector polarization) with the spin tracking code Spink.
\end{abstract}

\section{Spink}

Spink is a spin tracking code through the lattice of a synchrotron[2],[3]. In this code each machine element is treated as "thin" for spin motion and the spin angle rotation $\mu$ is then calculated by a "flattened" version of the BMT spin equation[4]

$$
\frac{d \mathbf{S}}{d t}=\frac{q}{\gamma m} \mathbf{S} \times \mathbf{F}
$$

with

$$
\mathbf{F}=(1+G \gamma) \mathbf{B}_{\perp}+(1+G) \mathbf{B}_{\|}
$$

containing the magnetic field components transverse and longitudinal with respect to the velocity $\mathbf{v}$, where $G$ is the particle gyromagnetic ratio (=-0.142987 for deuterons) and $\gamma$ the Lorentz factor for the energy.. The spin rotation is calculated along the particle orbit, while the magnetic field that appear in the equations are calculated in the laboratory reference frame.

The vector spin is treated as a 3-dimension real vector. spink uses "synchrotron" coordinates, defined as: $x$, radial, $y$, vertical, and $z$, longitudinal.

\section{RF Dipole simulation}

In the COSY experiment that we wanted to simulate, an RF Dipole was used, that produced an horizontal oscillating magnetic field at a location of the beam trajectory. The RF field gives a vertical periodic kick to the orbit and produces a proportional kick to the deuteron spin. The orbit kick modulates the vertical betatron motion of the beam. When the frequency of modulation of the orbit and the frequency of spin precession (spin tune) match through the condition

$$
f_{R F}=f_{c}(k \pm G \gamma),
$$

where $f_{R F}$ is the frequency of the RF, $f_{c}$ the frequency of circulation of the beam in the ring, $G \gamma$ the spin tune, or the relative frequency of spin precession in the machine, and $k$ is an integer, the spin flips from its direction (originally vertical) to a direction closer to the horizontal.

To obtain this flip, the RF of the Dipole was linearly varied in time, while the momentum of the deuteron beam was kept constant, until the condition (3) was met, with $k=1$. 
The expression for the kick imparted to the vertical momentum $p_{y}$ used in Spink is

$$
K=\frac{1}{B \rho}\left(\int B_{R F} d \ell\right) \cos \phi, \quad \phi=2 \pi \int \nu_{m}(t) d t, \quad \frac{\delta p_{y}}{p}=K,
$$

with $\nu_{m}$ a linear function of time. $B \rho=1.10^{9} \mathrm{pc} / \mathrm{c}$ the momentum of the beam, expressed in units of [Tesla.m], where $p c$ is expressed in [GeV]. $\int B_{R F} d \ell$ is the strength of the Dipole, also in [T.m]. $p_{y}$ is the vertical momemtum of a deuteron.

The spin precession kick by the RF dipole is simply expressed in Spink as

$$
\mu=G \gamma K
$$

The spin matrix for the rotation of the vector spin in the RF Dipole is (rotation around an horizontal axis, to first order in the components of the particle velocity)

$$
\left(\begin{array}{ccc}
1 & 0 & 0 \\
0 & \cos \mu & \sin \mu \\
0 & -\sin \mu & \cos \mu
\end{array}\right) .
$$

The result that leads to Eq.(5) to first order is obtained from the BMT equation integrated through the thin RF Dipole, when the vertical oscillations of the beam are taking into account. This results is in agreement with a recent note by E.Courant[5], submitted for publication.

With Spink we simulated the results of the cited SPINK@COSY article, in particular the curves of Fig.2 (measured vector deuteron polarization for different speed of crossing the resonance), and fig.4 (resonance strength for different vertical betatron tune of COSY).

We tracked in the same condition of the experiment, at constant momentum of the deuterons, namely

$$
p c=1.85[\mathrm{GeV}]
$$

and with an RF Dipole strenght

$$
B_{R F} d \ell=6.10^{-4}[\mathrm{~T} . \mathrm{m}] .
$$

The speed of crossing is defined as

$$
\alpha=\frac{\Delta f_{R F}}{\Delta t}[\mathrm{~Hz} / \mathrm{sec}]
$$

where the experiment used a fixed $\Delta f_{R F}=300[\mathrm{~Hz}]$.and $\Delta t$ was varied. The spin showed a flipping at the required frequency, Eq.(3). The vertical vector spin component behaves as shown in Fig.1

Since this is a well isolated spin resonance, we can calculate its strength $\epsilon_{F S}$ using the FroissartStora formula $[6]$

$$
P_{f}=P_{0}\left\{\exp \left[-\frac{\left(\pi \epsilon_{R F}\right)^{2}}{\alpha^{2}}\right]-1\right\} .
$$

Note that Spink does NOT use any pre-calculated formula to predict the resonance strength $\epsilon_{R F}$, which is only calculated from the ratio $P_{f} / P_{0}$,

\section{Results}

We first simulated by Spink tracking the experimental results summarized in Fg.2 of the COSY paper, i.e. resonance strength vs. speed of crossing, using Eq.(5) for the spin kick. The results are in our Fig.2 The black circles are the measured values and the red squares the results of Spink. Another set of data is shown in the figure with blue diamonds, obtained from a simulation where the spin kick was made (incorretly) proportional to $1+G \gamma$. Since for the deuteron momentum used in the experiment the coefficient for the spin kick is either the spin tune $G \gamma=-0.20084$ or $(1+G \gamma)=0.79916$, the results would differ by a factor 4 (hence the title of the paper), Comparison with the experiment shows that the first value for the coefficient is right and the second is wrong, in agreement with the cited Courant paper. 


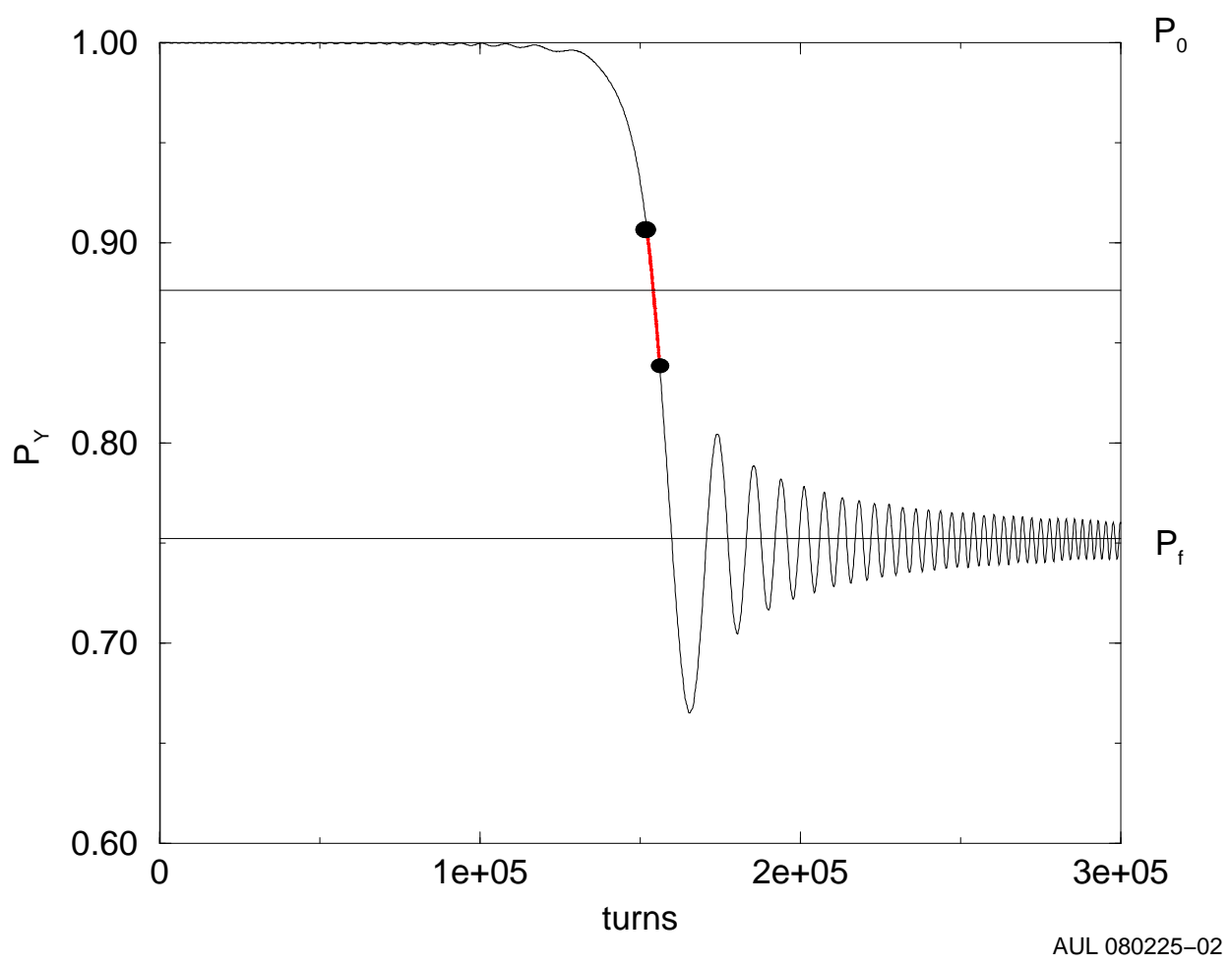

Figure 1: Example of spin flip. Vertical component of the polarization vs. turn number in COSY. The vertical betratron tune for this example was $\nu_{y}=3.783$. The horizontal lines mark the final polarization $P_{f}$ and the value of the polarization in the center of the resonance. 


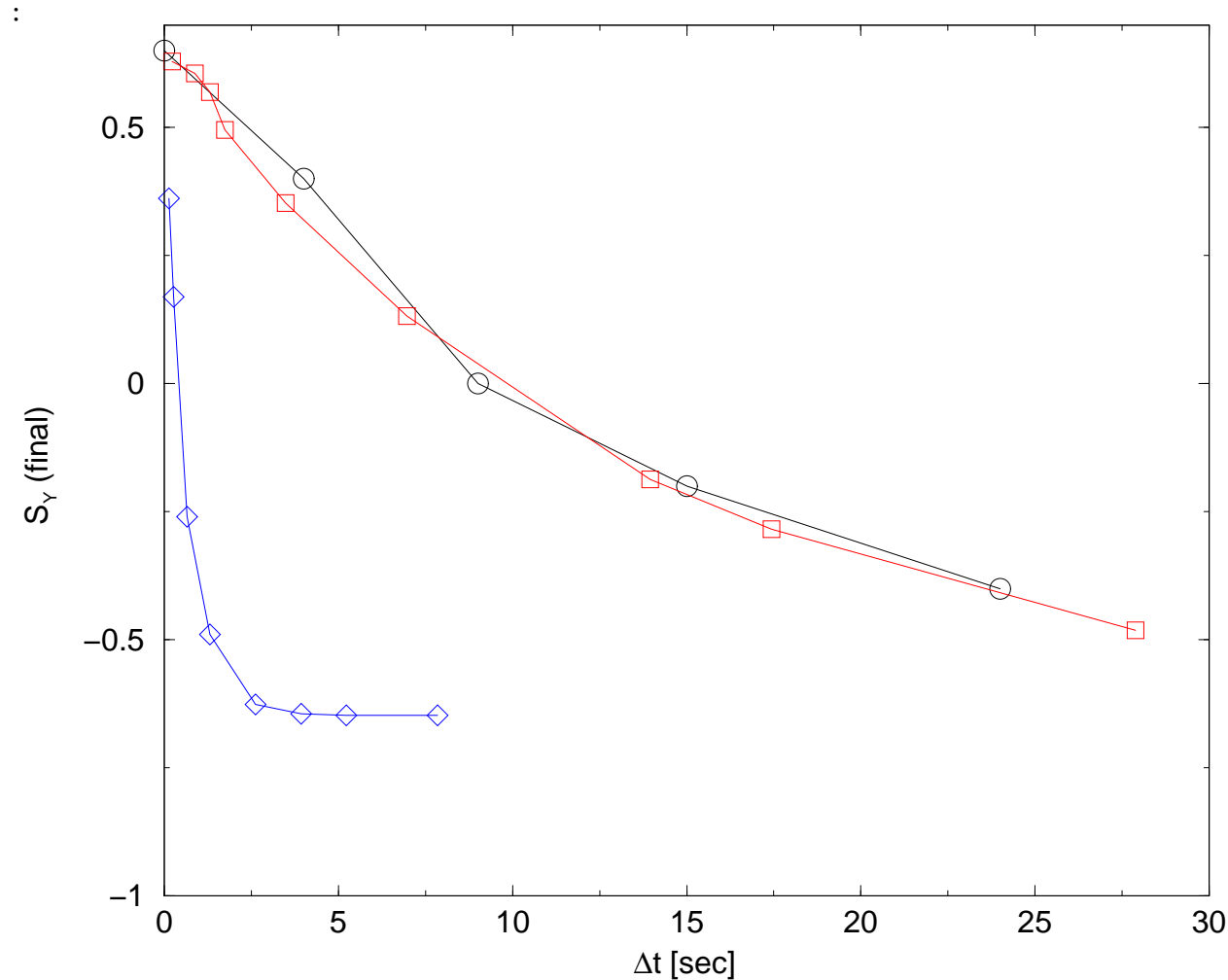

Figure 2: Comparison between Spink simulation and measured values for polarized deuterons resonanne induced by a RF Dipole. Circles indicate measured values for vector polarization, squares are values simulated with a $G \gamma$ coefficient in the RF Dipole, diamonds with $(1+G \gamma)$. The vertical axis is the vertical component of the spin, the horizontal axis is the duration of the RFD action, inversely proportional to the speed of resonance crossing. 


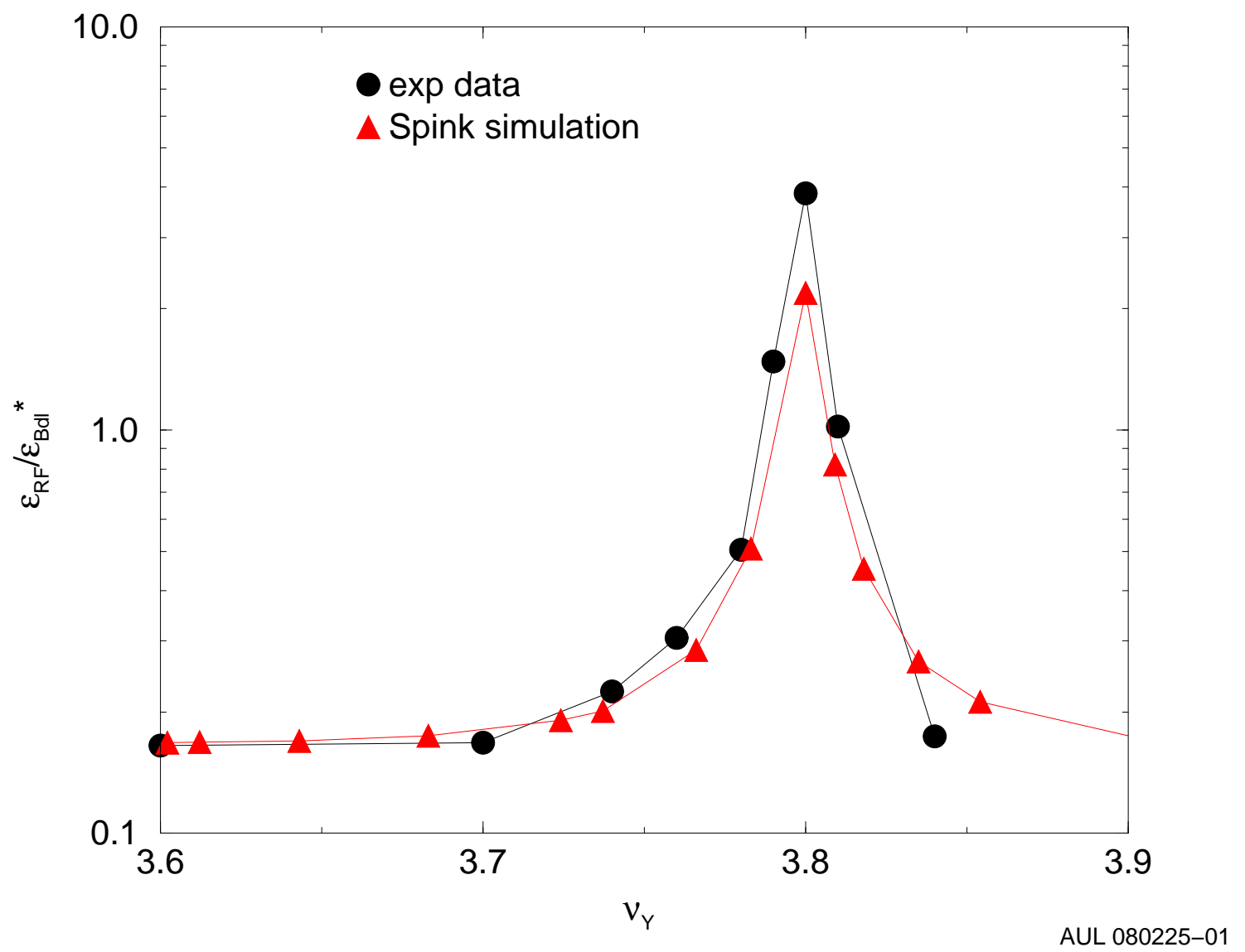

Figure 3: Comparison with Fig.3 of the quoted article. Black circles: experimental data, normalized. Red triangles: Spink simulation. 

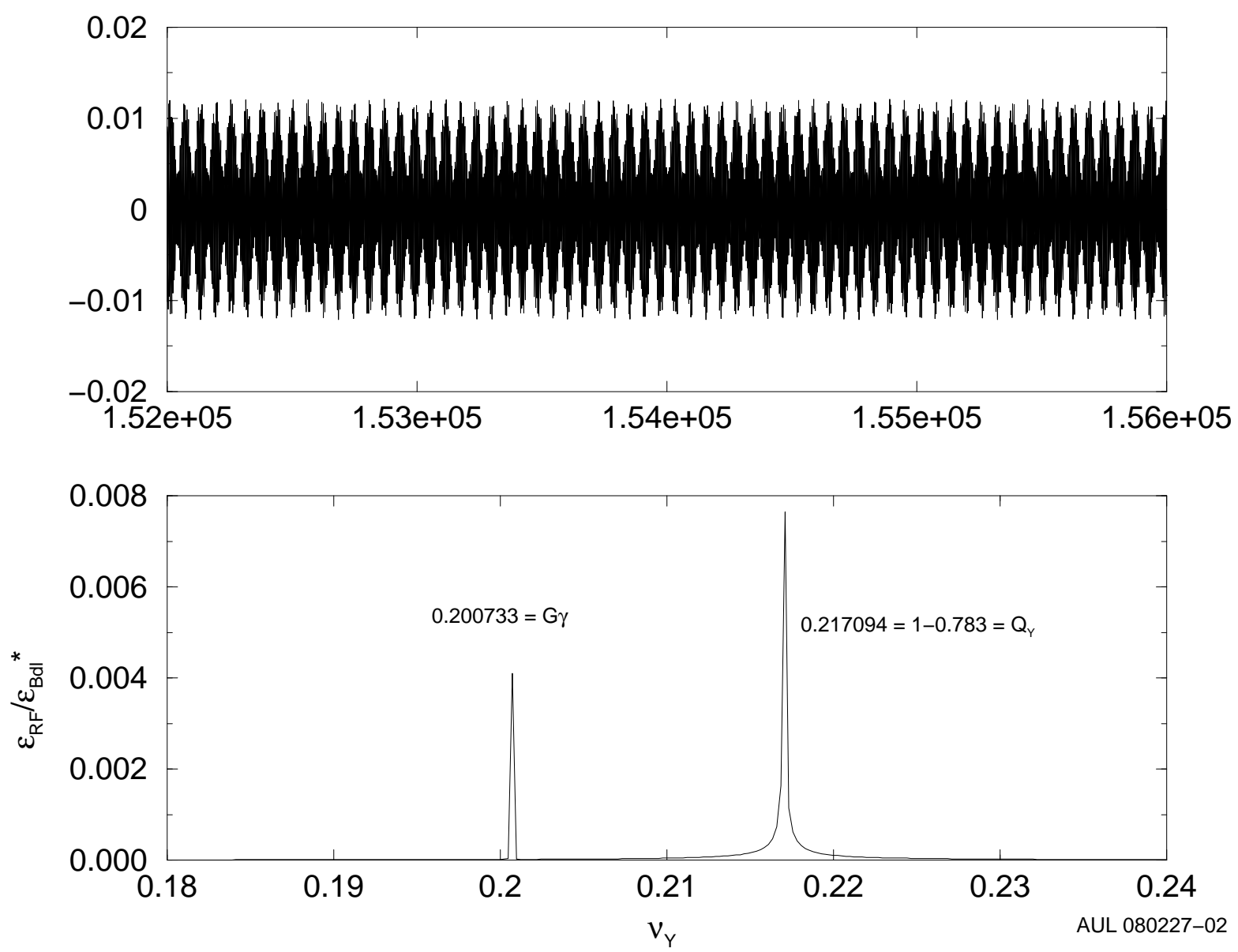

Figure 4: Vertical orbit vs. turn number (upper curve), and FFT spectrum (lower)

The measurements shown in Fig.4 of the COSY paper, resonance strength vs. COSY betatron tune, were also simulated with the results shown in our Fig.3. This figure shows the measured resonance strenght, normalized to a theoretical value. Here, at variance with Fig.2 the curve is always the same, whichever coefficient would be used in the formula for the spin kick, since the simulation results are proportional to that coefficient. In other words: this curve cannot be used to verify the correctness of the spin kick coefficient in the RF Dipole.

The vertical kick on the orbit by the RF Dipole modulates the vertical betatron oscillation. An FFT spectrum of the vertical orbit shows both the betatron tune line and the frequency of the modulation. The modulation spectral line moves during the RF ramp and coincides with the resonant frequency of Eq.(3) when the deuteron spin actually flips. This is reported in Fig.4 that shows the vertical orbit in the turn interval highlitened in Fig.1 and its spectrum. 


\section{Acknowledgments}

Discussions with E.D.Courant, Thomas Roser, Waldo MacKay, Vahid Ranjbar have been important to write this note.

\section{References}

[1] A.D.Krisch, M.A.Leonova, V.S.Morozov R.S.Raymond.D.W.Sivers V.K.Wong R.Gebel A Lehrach B.Lorentz R.Maier D.Prasuhn H.Stockhorst F.Hinterberger and K.UlBRICH: Unexpected reduction of rf spin resonance strength for stored deuteron beams. Phys.Rev.Spec.Topics-Accel.and Beams, (10):07100-1, 2007.

[2] A.U.Luccio: Spin Tracking in RHIC-Code SPINK. In: Y.Onel, N.PAVer, A.Penzo, editor: Proc. Adriatico Research Conf on Trends in Collider Spin Physics. Trieste, Italy, 12/5-8, 1995. World Scientific, p.235.

[3] A.U.Luccio: Spink User's Manual. Version v.2.21-beta. Technical Report C-A/AP/283, Brookhaven National Laboratory. Upton, NY, August 2007.

[4] D.J.JAckson: Classical Electrodynamics. Wiley, New York, 1975. Second edition.

[5] E.D.Courant: Spin Motion and Resonances in Accelerators and Storage Rings. Technical Report C-A/AP/293, Brookhaven National Laboratory. Upton, NY, Upton, NY, unpublished.

[6] M.Froissart and R.Stora. Nuclear Inst. and Meth., (7):297, 1960. 